THE ASTROPHYSICAL JouRnaL, 337:917-926, 1989 February 15

(C) 1989. The American Astronomical Society. All rights reserved. Printed in U.S.A.

\title{
ON THE STABILITY OF DECELERATING SHOCKS
}

\author{
ETHAN T. VishNiaC AND DONGSU RYU \\ Department of Astronomy, University of Texas \\ Received 1988 June 1; accepted 1988 August 3
}

\begin{abstract}
Previous work has shown that when the high-density region behind a decelerating pressure-driven shock wave is sufficiently thin, then the shock wave is subject to a rippling instability. Here we give an analytic analysis of this instability for a decelerating isothermal shock wave including terms that suppress the instability at short wavelengths. We use the resulting dispersion relation to suggest approximate formulae governing the dynamical evolution of the high-density region behind a shock wave. This allows us to calculate the maximum growth rate as a function of wavelength for an arbitrary shock. We compare our results to exact calculations of the evolution of linear perturbations of adiabatic shocks. Finally, we predict that isothermal blast waves with postshock density contrasts greater than $\sim 10$ will be unstable. Wind-driven isothermal shocks are more stable with a critical density contrast of $\sim 25$. We conclude that a fair description of the hydrodynamic stability of shocks is possible without reference to the details of their postshock vertical structure.
\end{abstract}

Subject headings: hydrodynamics — shock waves

\section{INTRODUCTION}

The stability of hydrodynamic shock waves is a problem of long-standing interest to physicists and astrophysicists alike. Erpenbeck (1962) showed that a shock wave, idealized as the boundary between two semi-infinite volumes with uniform conditions throughout, would be stable for a very broad range of fluid properties. More realistically we expect that the postshock fluid will have a complicated distribution of density and pressure which will affect its stability. An obvious example is the case where the shock is driven by the accelerating expansion of a hot fluid. In this case the density of the postshock material actually rises as one passes from the shock wave toward the interior. The interface between the hot, interior fluid and the postshock layer appears strongly similar to the classic illustration of a Rayleigh-Taylor instability. Consequently, the proof by Bernstein and Book (1978) that this interface is unstable is intuitively appealing. This instability may be applicable in a number of astrophysical situations, most notably those where a pressure-driven shock is propagating down a steep density gradient. However, the case of the decelerating shock wave, typical of a shock propagating in a uniform density environment, is more complicated.

Vishniac (1983, hereafter V83) analyzed the case of a shell of gas bounded on the outside by ram pressure and on the inside by a gas with finite pressure and infinitesimal density. His analysis was limited by the assumption that the perturbation wavelength was much greater than the thickness of the postshock layer. This work revealed a nonradial instability, actually a growing oscillation, present on scales intermediate between the radius of the blast wave and the thickness of the postshock layer. If the two scales are not sufficiently distinct then no instability is present. This result was confirmed, using a somewhat different approach, by Bertschinger (1986). The minimum unstable wavelength cannot be recovered from this work. It is clear from Erpenbeck's analysis that this instability must be suppressed on sufficiently small scales, but the thin shell approximation includes neglecting all terms of order $H / R$, where $H$ is the thickness of the postshock layer and $R$ is the radius of the blast wave. (For cases without spherical sym- metry the radius may be replaced, up to a constant of order unity, by the distance the shock will propagate before its characteristics change significantly.)

Subsequently, Ryu and Vishniac $(1987,1988)$ have derived the eigenvalues and eigenfunctions for nonradial linear perturbations of the Sedov solution and the wind-driven shock wave. These results clearly show the small wavelength suppression of the instability. Furthermore, they show that the wavelength of maximum instability will, in both cases, be about twice the minimum unstable wavelength. The latter result suggests that there are features of the small wavelength stabilization that are independent of the particular details of the shock wave in question. A general theory for shock wave rippling must exist which includes these features.

In this paper we will present an approximate analysis of the postshock layer in the case where the postshock gas is isothermal. We present this work not only to extend the number of cases where the linear overstability has been shown to be present, but also to suggest an approximate dispersion relation which can be applied to shocks with arbitrary postshock structure. We wish to stress the following four points. First, for perturbation wavelengths much greater than the shell thickness our results reduce to the thin shell analysis. Second, we have neglected terms of order $H / R$, but not terms of order $H / \lambda$, where $\lambda$ is the perturbation wavelength. Third, this work does not include the internal degrees of freedom of the dense layer. Although the layer is not held to a uniform thickness, sound waves within the layer are explicity discarded unless they are fundamental radial modes of the postshock gas layer. Fourth, this work will apply to a broad class of shock waves. Nevertheless, some possible complications, such as magnetic fields, are explicitly neglected. Others, such as the details of the cooling flow in a radiative region, are allowed for only as corrections to the averaged parameters of the dense layer.

In $\S$ II we analyze the short-wavelength linear perturbations of the postshock gas assuming isothermality. Effects which are important at longer wavelengths are included by analogy with the thin shell case. We derive some simple results from this including the maximum growth rate, and the minimum 
unstable wavelength. In $\S$ III we find exact solutions to these equations for the case of a self-similar shock wave in a uniform medium. In particular, we compare the results of this analysis to the exact solutions for the Sedov solution and the winddriven shock wave (Ryu and Vishniac 1987, 1988). We summarize our results in $\S \mathrm{IV}$.

\section{ANALYSIS}

We start by considering a planar isothermal shock located at $z=0$ with a dense layer of postshock gas confined in the range $0<z<H$. At $z=0$ the confinement is caused by a stream of exterior gas with velocity $V_{s}$ and density $\rho_{E}$. At $z=H$ the gas is bounded by interior gas of infinitesimal density but finite thermal pressure $\boldsymbol{P}_{i}$. The gas is subject to an acceleration, $\dot{V}_{\boldsymbol{s}}$, which on short time scales is equivalent to applying a gravitational acceleration in the $-\hat{z}$ direction. Strictly speaking these conditions cannot be satisfied exactly. For example, if the acceleration is constant and the shock always occurs at $z=0$ then the ram pressure $\rho_{E} V_{s}^{2}$ must be steadily increasing (or the pressure $P_{i}$ must be steadily decreasing). In our analysis we will neglect changes in the structure of the shock. In practice this means restricting ourselves to short time scales or frequencies $\omega$ such that $\omega \tau \gg 1$, where $\tau$ is the evolutionary time scale of the shock.

Expanding in powers of the shock Mach number, $\mathscr{M} \equiv$ $V_{s} / c_{s}$, we get as a first approximation

$$
\rho(z)=\rho_{0} e^{-z / L},
$$

and

$$
\rho_{0}=\rho_{E} V_{s}^{2} / c_{s}^{2},
$$

where $L \equiv c_{s}{ }^{2} /\left|\dot{V}_{s}\right|$ is the scale height of the gas, and $c_{s}$ is the sound speed. In order to maintain the density law given in equation (1) there will, in general, be some internal velocity field $u_{0}(z)$. Its value at some arbitrary $z$ depends on the long term evolution of the shock, but its value at $z=0$ must be $c_{s}^{2} / V_{s}$ from the shock jump conditions. We note that as a consequence of these definitions and equations (1) the thickness of the layer, $H$, and the column density, $\sigma$, are given by

$$
H=L \ln (1 / \beta) \text {, }
$$

and

$$
\sigma=\rho_{0} L(1-\beta)
$$

where

$$
\beta \equiv \frac{P_{i}}{\rho_{E} V_{s}^{2}} .
$$

Now suppose we assume that the shock suffers a linear perturbation proportional to $e^{i(\omega t+k x)}$, where $\hat{x}$ is perpendicular to $\hat{z}$. The perturbation variables are the fractional density perturbation, $\delta \equiv \delta \rho / \rho$, and the velocity perturbation, $v \equiv v_{x} \hat{x}+v_{z} \hat{z}$. Assuming the gas is strictly isothermal the evolution of the linear perturbation is governed by three equations, i.e.,

$$
\begin{gathered}
\left(\delta \partial_{z} u_{z}\right)+\left[i \omega+\left(u_{z} \partial_{z}\right)\right] \delta+i k v_{x}+\partial_{z} v_{z}-v_{z} / L=0 \\
{\left[i \omega+\left(u_{z} \partial_{z}\right)\right] v_{x}+i k c_{s}^{2} \partial=0}
\end{gathered}
$$

and

$$
\left[i \omega+\left(u_{z} \partial_{z}\right)\right] v_{z}+c_{s}^{2} \partial_{z} \delta+\left(v_{z} \partial_{z} u_{z}\right)=0 .
$$

The terms in parentheses are small provided that $\partial_{z} \sim L^{-1}$.
This is certainly true when considering terms like $\partial_{z} u_{z}$. It is not always true when considering the gradient of the perturbation variables.

Let's assume that we can ignore the terms in parentheses. We find two independent solutions given by

$$
\begin{gathered}
\delta=B_{ \pm} \exp \left(\lambda_{ \pm} z\right) \\
\lambda_{ \pm}=\frac{1}{2 L} \pm\left(\frac{1}{4 L^{2}}+k^{2}-\frac{\omega^{2}}{c_{s}^{2}}\right)^{1 / 2}, \\
v_{x}=-\frac{k}{\omega} c_{s}^{2} \delta
\end{gathered}
$$

and

$$
v_{y}=i \frac{\lambda_{ \pm}}{\omega} c_{s}^{2} \delta
$$

In order for these solutions to be self-consistent we need to ignore solutions where $\omega / c_{s}>k$ and $\omega / c_{s} \gg 1 / L$. One obvious example of such a solution is a high-frequency sound wave whose wave vector is not aligned with the shock front.

Equations (3) are a third-order set of equations in $z$ for which we have found two (approximate) solutions. In order to find the third solution we must assume that it has large gradient at some $z$ so that the $u_{z} \partial_{z}$ terms can be important. By inspection we see that this third solution is

$$
\begin{gathered}
v_{x}=A \exp \left(-i \int \frac{\omega d z}{u_{z}}\right), \\
v_{z}=\frac{A u_{z} k}{\omega} \exp \left(-i \int \frac{\omega d z}{u_{z}}\right),
\end{gathered}
$$

and

$$
\delta=0
$$

If the perturbation mode is unstable then this solution will fall rapidly to zero away from $z=0$. We will assume this is the case and take $u_{z}=u_{z}(0)$ in what follows.

The boundary conditions at the shock front are

$$
v_{x}(0)=V_{s} \nabla \Delta z(0)=V_{s} \frac{k}{\omega} v_{z}(0),
$$

and

$$
\delta(0)=\frac{\Delta z(0)}{L}-\frac{2 v_{z}(0)}{V_{s}} \approx \frac{v_{z}(0)}{i \omega L} .
$$

In equations ( $6 \mathrm{a})$ and $(6 \mathrm{~b}), \Delta z(0) \approx v_{z}(0) / i \omega$ is the displacement of the shock surface away from $z=0$. The second term on the right-hand side of equation (6b) comes from the change in the ram pressure due to the motion of the shock surface. It can be neglected in favor of the first term because we expect that $\omega$ is only of order $c_{s} / L$ or less. The boundary condition at the pressure-bounded surface $(z=H)$ is

$$
\delta(H)=\frac{\Delta z(H)}{L}=\frac{v_{z}(H)}{i \omega L},
$$

which arises from the condition that the pressure at the composition discontinuity is always $\boldsymbol{P}_{i}$.

We obtain our dispersion relation by substituting equations (4) and (5) into equations (6) and (7). Starting with equation (7) 
we obtain

$$
B_{+} \beta^{-Q}\left(1-\frac{c_{s}{ }^{2} \lambda_{+}}{\omega^{2} L}\right)+B_{-}\left(1-\frac{c_{s}{ }^{2} \lambda_{-}}{\omega^{2} L}\right)=0,
$$

where

$$
Q \equiv\left[1+(k L)^{2}-\left(\omega L / c_{s}\right)^{2}\right]^{1 / 2} .
$$

Equations (6) imply

$A\left[1-\left(\frac{k c_{s}}{\omega}\right)^{2}\right]=\frac{k c_{s}^{2}}{\omega}\left[B_{+}\left(1+\frac{i V_{s} \lambda_{+}}{\omega}\right)+B_{-}\left(1+\frac{i V_{s} \lambda_{-}}{\omega}\right)\right]$,

and

$$
B_{+}\left(1-\frac{c_{s}^{2} \lambda_{+}}{L \omega^{2}}\right)+B_{-}\left(1-\frac{c_{s}^{2} \lambda_{-}}{L \omega^{2}}\right)=A \frac{k c_{s}^{2}}{i V_{s} \omega^{2} L}
$$

Before proceeding further it is worthwhile considering the magnitude of $i V_{s} \lambda_{ \pm} / \omega$, i.e., the second term in the coefficient of $B_{ \pm}$in equation (9a). In the large frequency limit $\lambda_{ \pm} \sim \omega / c_{s}$. This implies that this term is of order $V_{s} / c_{s} \gg 1$. In the lowfrequency limit this term can be minimized by choosing to consider $\lambda_{-}$. In that case

$$
i V_{s} \lambda_{ \pm} / \omega \sim i V_{s} \omega L / c_{s}^{2} \sim V_{s} \omega / \dot{V}_{s} \gg 1 .
$$

This last expression is required to be large for our analysis to be self-consistent. In either limit we see that this term is much larger than 1. This allows us to rewrite equation (9a) as

$$
A\left[1-\left(\frac{k c_{s}}{\omega}\right)^{2}\right]=\frac{i V_{s} k c_{s}^{2}}{\omega^{2}}\left(B_{+} \lambda_{+}+B_{-} \lambda_{-}\right),
$$

We can eliminate $A$ by combining equations (9b) and (11). We obtain

$$
B_{+}\left(1-\frac{k^{2} c_{s}{ }^{2}}{\omega^{2}}-\frac{c_{s}{ }^{2} \lambda_{+}}{L \omega^{2}}\right)+B_{-}\left(1-\frac{k^{2} c_{s}{ }^{2}}{\omega^{2}}-\frac{c_{s}{ }^{2} \lambda_{-}}{L \omega^{2}}\right)=0 .
$$

We can combine equations (8) and (12) and use $\lambda_{+} \lambda_{-}=$ $\left(\omega^{2} / c_{s}{ }^{2}-k^{2}\right)$, and $\lambda_{+}+\lambda_{-}=1 / L$ to obtain (after some manipulation)

$$
\omega^{4}-c_{s}^{2} \omega^{2} k^{2}+\frac{k^{2} c_{s}^{4}}{2 L^{2}}\left(\frac{1+\beta^{Q}}{1-\beta^{Q}} Q-1\right)=0,
$$

or

$$
\omega^{4}-c_{s}^{2} \omega^{2} k^{2}+\frac{k^{2} \dot{V}_{s} P_{i}}{\sigma} F(\beta, Q)=0
$$

where

$$
F(\beta, Q)=\frac{1-\beta}{2 \beta}\left(\frac{1+\beta^{Q}}{1-\beta^{Q}} Q-1\right)
$$

and $Q$ is given in equation ( $8 \mathrm{~b})$. We note that as $Q \rightarrow 1, F(\beta, Q)$ $\rightarrow 1$. This dispersion relation describes the linear modes of an isothermal shock ignoring all effects having to do with the evolution of the shock as a whole. We can include such effects by comparing the results of earlier work using the thin shell approximation (V83).
In the thin shell approximation the equations governing the evolution of a spherical shock wave are

$$
\begin{gathered}
\partial_{t} \sigma=-2\left(V_{s} / R_{s}\right) \sigma+\rho_{E} V_{s}-\sigma\left(\nabla_{T} \cdot V_{T}\right), \\
\partial_{t} V_{s}=-\sigma^{-1}\left(\rho_{E} V_{s}^{2}-P_{i}\right),
\end{gathered}
$$

and

$$
\partial_{t} V_{T}=-\left(\frac{\rho_{E} V_{s}}{\sigma}\right) V_{T}-\left(\frac{V_{s}}{R_{s}}\right) V_{T}-\frac{\left\langle c_{s}^{2}\right\rangle}{\sigma} \nabla_{T} \sigma-\frac{P_{i}}{\sigma} \nabla_{T} R_{s},
$$

where $\sigma$ is the surface density of the postshock gas, $\rho_{E}$ is the density of the exterior gas, $P_{i}$ is the pressure supplied by the hot, diffuse, interior gas, $V_{T}$ is the tangential gas velocity, and $R_{s}$ and $V_{s}$ are average radial position and velocity of the gas. The subscript $\boldsymbol{T}$ refers to gradients and divergences taken only in the $\theta$ and $\phi$ directions. Strictly speaking we should make a distinction between the position and velocity of the shock front and the average position and velocity of the gas, but this only introduces correction terms of order $H / R$ regardless of the perturbation wavelength. These equations refer only to the case of a spherical shock, but can be trivially modified to reflect other symmetries by taking $R_{s}$ to be the local radius of curvature of the shock front.

In order to evaluate the stability of the shock front we defined perturbation variables $\Delta R \equiv R_{s}-R_{0}, \delta \equiv \sigma / \sigma_{0}-1$, and $\Delta z \equiv R_{s}-R_{i}$, where the subscript 0 denotes the unperturbed solution. The quantity $V_{T}$ is already a perturbation to pure radial expansion. Equations (14) then become

$$
\begin{gathered}
\partial_{t} \delta=\frac{\partial_{t} \Delta R}{R_{0}}+\frac{2 V_{s} \Delta R}{R_{0}^{2}}-\frac{\rho_{E} V_{s} \delta}{\sigma_{0}}-\nabla_{T} \cdot V_{T}, \\
\partial_{t}{ }^{2} \Delta R=-\dot{V}_{s} \delta-2 \frac{\rho_{E} V_{s}}{\sigma_{0}} \partial_{t} \Delta R,
\end{gathered}
$$

and

$$
\partial_{t} V_{T}=-\frac{V_{s}}{R_{s}} V_{T}-\frac{\rho_{E} V_{s}}{\sigma_{0}} V_{T}-\frac{P_{i}}{\sigma_{0}} \nabla_{T} \Delta R-\left\langle c_{s}^{2}\right\rangle \nabla_{T} \delta .
$$

The last term on the right-hand side of equation $(15 \mathrm{c})$ was shown to be small compared to the others provided that $k H \ll 1$ (V83).

Ignoring the terms which describe the evolution of the background solution we see that the dispersion relation for linear perturbations with a transverse wavenumber $k$ and a frequency $\omega$ is

$$
\omega^{4}-\omega^{2} k^{2}\left\langle c_{s}{ }^{2}\right\rangle-\frac{P_{i} \dot{V}_{s} K^{2}}{\sigma_{0}}=0 .
$$

For a decelerating shock this implies the existence of two growing and two decaying oscillating solutions at long wavelengths. At short wavelengths we find sound waves and gravity waves. For an accelerating shock we have sound waves and one growing and one decaying mode.

Comparing equations (13) and (16) we see that abandoning the thin shell approximation has remarkably little effect on the dispersion relation. This is at least partly due to the fact that we have restricted our attention to perturbations that are fundamental modes of the postshock gas (in the $\hat{z}$ direction). The only sign of the internal dynamics comes from the presence of 
the function $F(\beta, Q)$ in equation (13). This suggests that a reasonable approach to the dynamics of the postshock gas is to use a slightly modified version of equations (15) in which we replace equation $(15 \mathrm{c})$ with

$$
\begin{aligned}
\partial_{t} V_{T}=-\frac{V_{s}}{R_{s}} V_{T}-\frac{\rho_{E} V_{s}}{\sigma_{0}} & V_{T} \\
& \quad-\frac{P_{i} F(\beta, Q)}{\sigma_{0}} \nabla_{T} \Delta R-\left\langle c_{s}{ }^{2}\right\rangle \nabla_{T} \delta .
\end{aligned}
$$

In equation (17) the value of $Q$ is somewhat ambiguous. It should be assigned a value on the basis of local definitions of $k$ and $\omega$.

Equation (16) implies that the wavelength of maximum growth is just twice the minimum unstable wavelength. The maximum growth rate is

$$
\Gamma_{\max }=\frac{1}{2}\left(\frac{-P_{i} \dot{V}_{s}}{c_{s}^{2} \sigma_{0}}\right)^{1 / 2},
$$

which occurs at a wavelength of

$$
\lambda_{\max }=2 \pi c_{s}^{2}\left(\frac{-P_{i} \dot{V}_{s}}{\sigma_{0}}\right)^{-1 / 2}
$$

Strictly speaking, this result only follows in the case $F(\beta, Q)$ $=1$, but at least it gives some insight into the relationship between the wavelength of maximum instability and the minimum unstable wavelength.

For any realistic shock we must include the stabilizing effect of the evolutionary terms neglected in equations (13) and (16). In general this will require integrating equations (15a), (15b), and (17). However, in certain idealized situations, it is possible to reduce the problem to a straightforward eigenvalue evaluation. We will discuss some particular examples in the next section.

\section{SELF-SIMILAR SHOCKS IN UNIFORM MEDIA}

Exact solutions can be derived for spherical shock waves with $R_{s} \propto t^{M}$ expanding into a uniform external medium. In this case the perturbations can be decomposed into spherical harmonics. For any particular value of $l$ the density perturbation grows as a power law in time, i.e., $\delta \propto t^{s}$. In this case equations (15a), (15b), and (17) can be reduced to a polynomial in $s$, i.e.

$$
\begin{gathered}
(s+3 M)(s+5 M-1)[(s+M)(s+7 M-1)-M(1-M)] \\
=-l(l+1)\left[(s+M)(s+7 M-1) \mathscr{M}^{-2} M^{2}\right. \\
\left.+\frac{M^{4} \beta F(\beta, Q)}{D^{2}(1-\beta) \mathscr{M}^{4}}\right],
\end{gathered}
$$

where $F(\beta, Q)$ is given by equation $(8 \mathrm{~b})$ and

$$
Q=\left(1+4 D^{2} l^{2}+4 \frac{D^{2} \mathscr{M}^{2}}{M^{2}} s^{2}\right)^{1 / 2} .
$$

In equations (19) $\mathscr{M}$ is the Mach number of the shock, and $D$ denotes the ratio $L / R_{s}$. The appropriate value of $\beta$ depends on $M$ and on the symmetry of the zeroth order solution. Assuming an infinitely thin shell we have $\beta=4 / 3-1 /(3 M)$. For a realistic shock $\beta$ will have some slightly smaller value which must be explicitly calculated. The value of $L$, and hence of $D$, is some- what ambiguous if the shock wave is not isothermal. Here we will assume that $L \rho_{s}=\sigma /(1-\beta)$, where $\rho_{s}$ is the postshock density. We also need $\mathscr{M}$ to solve equation (19), but this will depend on the particular physics of the shock wave.

Equation (19) can be used to find the condition for marginal stability, i.e., $s$ purely imaginary. After some manipulation we can show that this occurs when

$$
\begin{array}{r}
P^{2}\left(\frac{M^{2}}{\mathscr{M}^{2} 2}\right)^{2}+P\left[-M^{3}(1-3 M) \frac{3}{2 \mathscr{M}^{2}}-\frac{M^{4} \beta F\left(\beta, Q^{*}\right)}{D^{2} \mathscr{M}^{4}(1-\beta)}\right. \\
\left.+\frac{M^{2}}{2 \mathscr{M}^{2}}(8 M-1)^{2}\right]+ \\
+(8 M-1)^{2} \frac{M}{2}(23 M-5) \\
+(7 M-1)^{2}\left(\frac{M}{2}\right)^{2}=0,
\end{array}
$$

where

$$
Q^{*}=\left[1-\frac{2 \mathscr{M}^{2} D^{2}(23 M-5)}{M}+2 P D^{2}\right]^{1 / 2}
$$

and

$$
P \equiv l(l+1)
$$

We can use equations (20) to obtain the value of $\gamma$ for which a shock wave is marginally stable, i.e., the value for which the roots $P$ of equation (20a) are degenerate. Since $Q^{*}$ is a function of $P$ solutions to equation (20a) must be found through an iterative procedure. For the Sedov solution we have $M=0.4$, and

$$
\beta=\frac{2}{\gamma+1}\left(\frac{\gamma+1}{2 \gamma}\right)^{v_{1}} 2^{-\gamma /(2-\gamma)}\left[\frac{2(2 \gamma+1)}{7-\gamma}\right]^{v_{2}},
$$

where

$$
v_{1}=\frac{4 \gamma}{3 \gamma-1}
$$

and

$$
v_{2}=\frac{13 \gamma^{2}-7 \gamma+12}{(3 \gamma-1)(2-\gamma) 5}
$$

For adiabatic jump conditions we take

$$
\mathscr{M}^{-2}=2 \gamma(\gamma-1) /(\gamma+1)^{2},
$$

the value just behind the shock front. We can iterate to obtain the correct solution by taking an estimate of $\gamma$ to get $\beta(\gamma)$ in equation (20). We find the condition for marginal stability is $\gamma=1.197$. For $\gamma=1.1$ equations (20) suggest that the instability will be present for values of $l$ between 9.86 and 68.9. Numerical work (Ryu and Vishniac 1987) suggest marginal stability when $\gamma \approx 1.2$ and instability for $9.5<l<80$ when $\gamma=1.1$. A comparison between the values of $\Re s$ derived from equation (19) and numerical calculations for $\gamma=1.1,1.2$, and 1.4 is shown in Figure 1. We note that the agreement is reasonable for $\gamma=1.1$ or 1.2 and rather poor for $\gamma=1.4$. Of course, as $\gamma$ increases, $H / R$ increases, and we expect equation (19) to become less accurate. An additional source of difficulty is that we have used the value of the sound speed from directly behind the shock and the value of $\beta$ from the center of the blast wave. In reality the value of the sound speed rises as one crosses the dense shell moving inward, and the appropriate value of $\beta$ is 

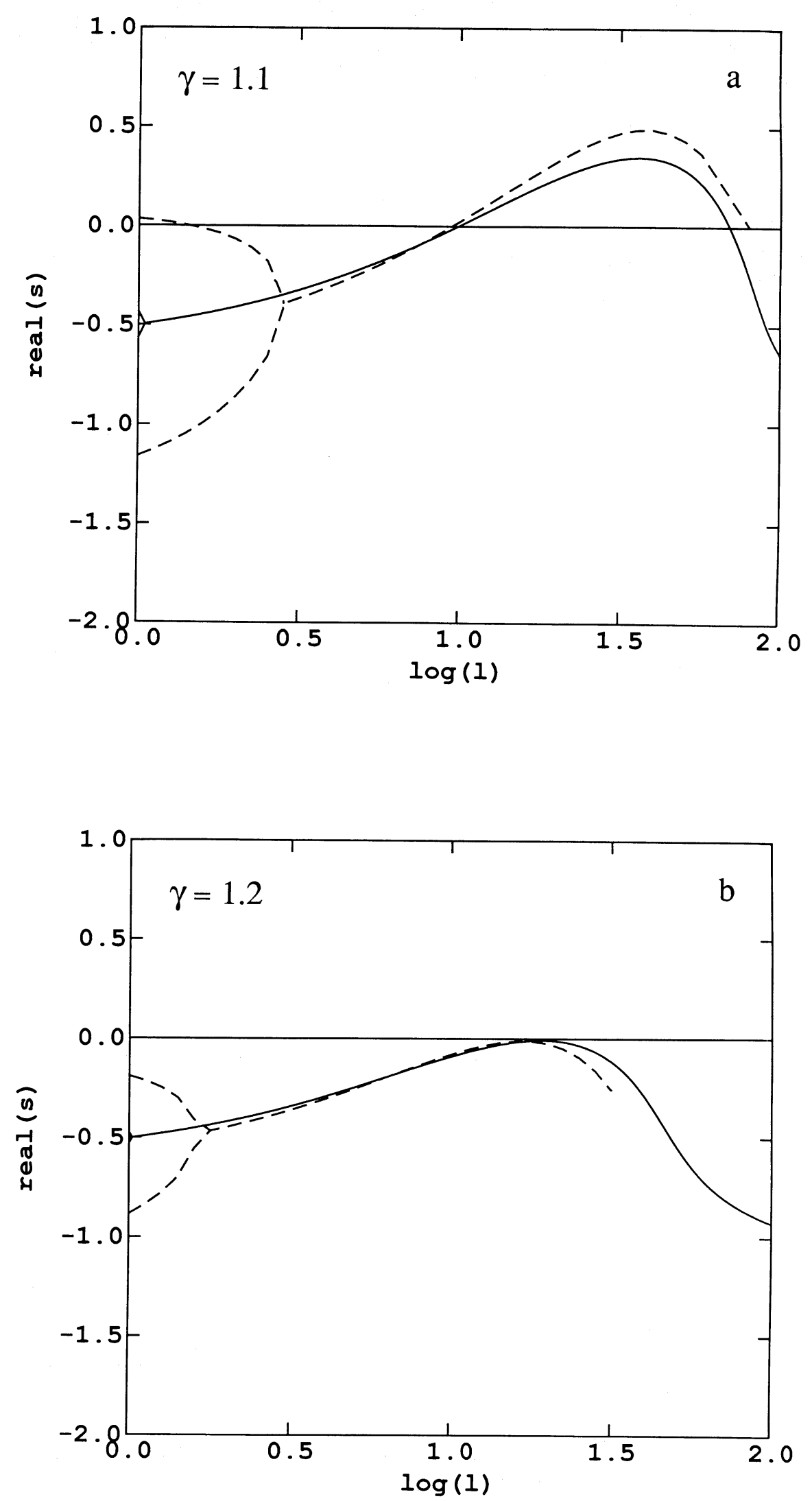

FIG. 1. $-(a-d)$ A plot of the dimensionless growth rate, real(s), as a function of spherical eigennumber for perturbations of an adiabatic blast wave. Shown are a comparison of the result of the isothermal approximation described in the text (solid line) with the results of an exact calculation (dashed line) for four different values of the adiabatic index. 

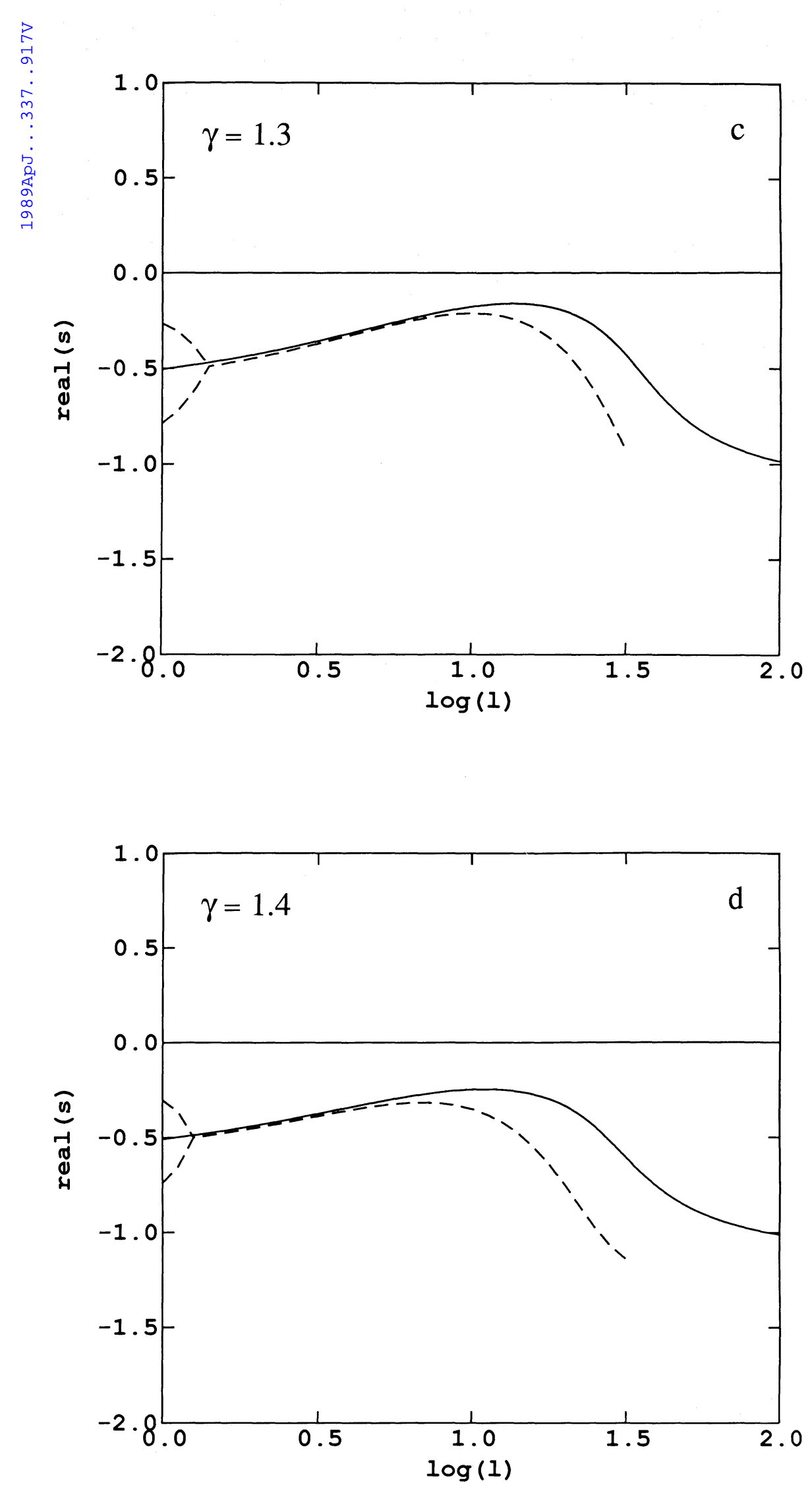

Fig. 1-Continued

922 

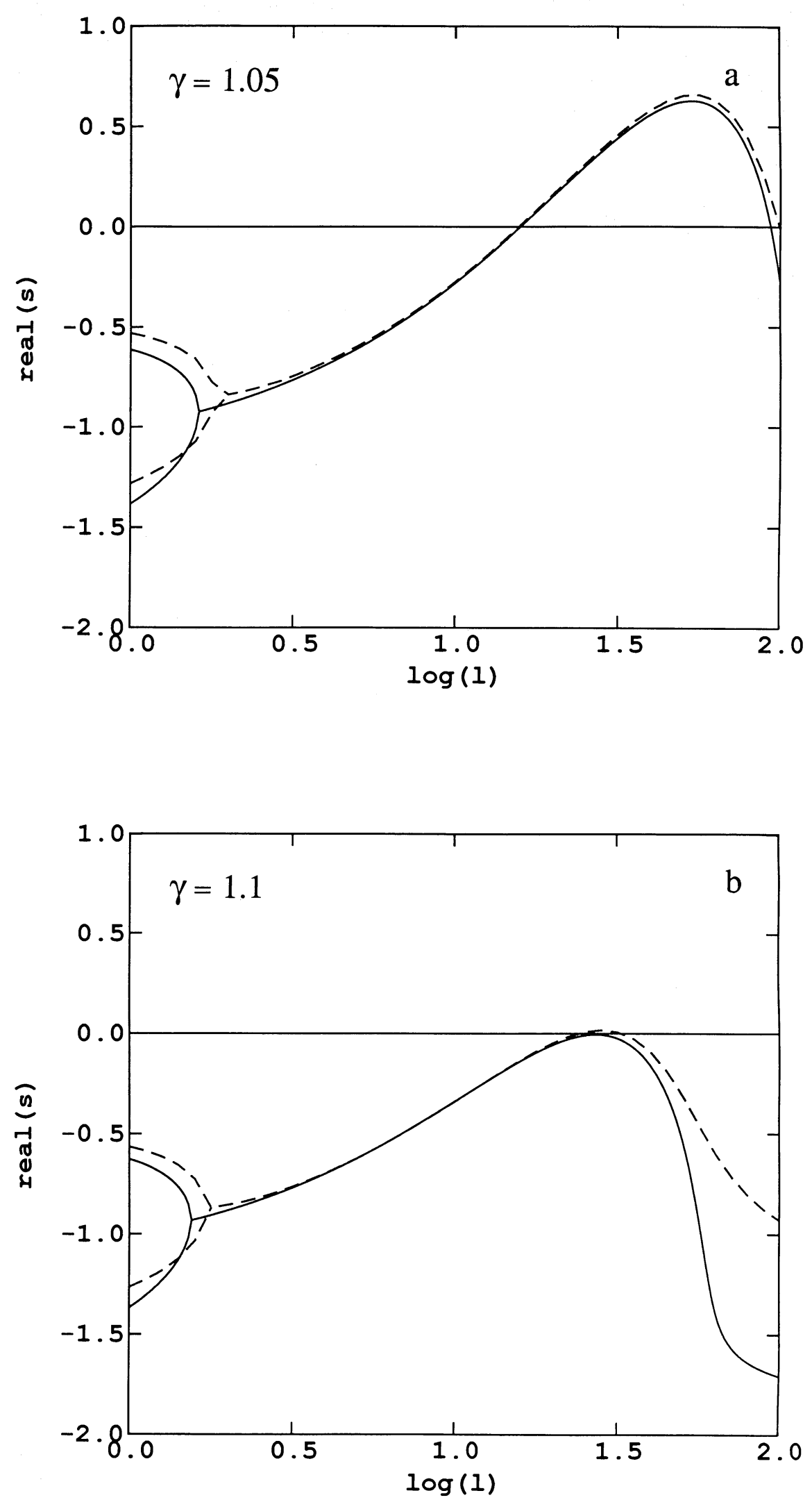

FIG. 2. - $(a-d)$ A plot of the dimensionless growth rate, real(s), as a function of spherical eigennumber for perturbations of an adiabatic wind driven shock. Shown are a comparison of the results of the isothermal approximation described in the text (solid line) with the results of an exact calculation (dashed line) for four different values of the adiabatic index. 

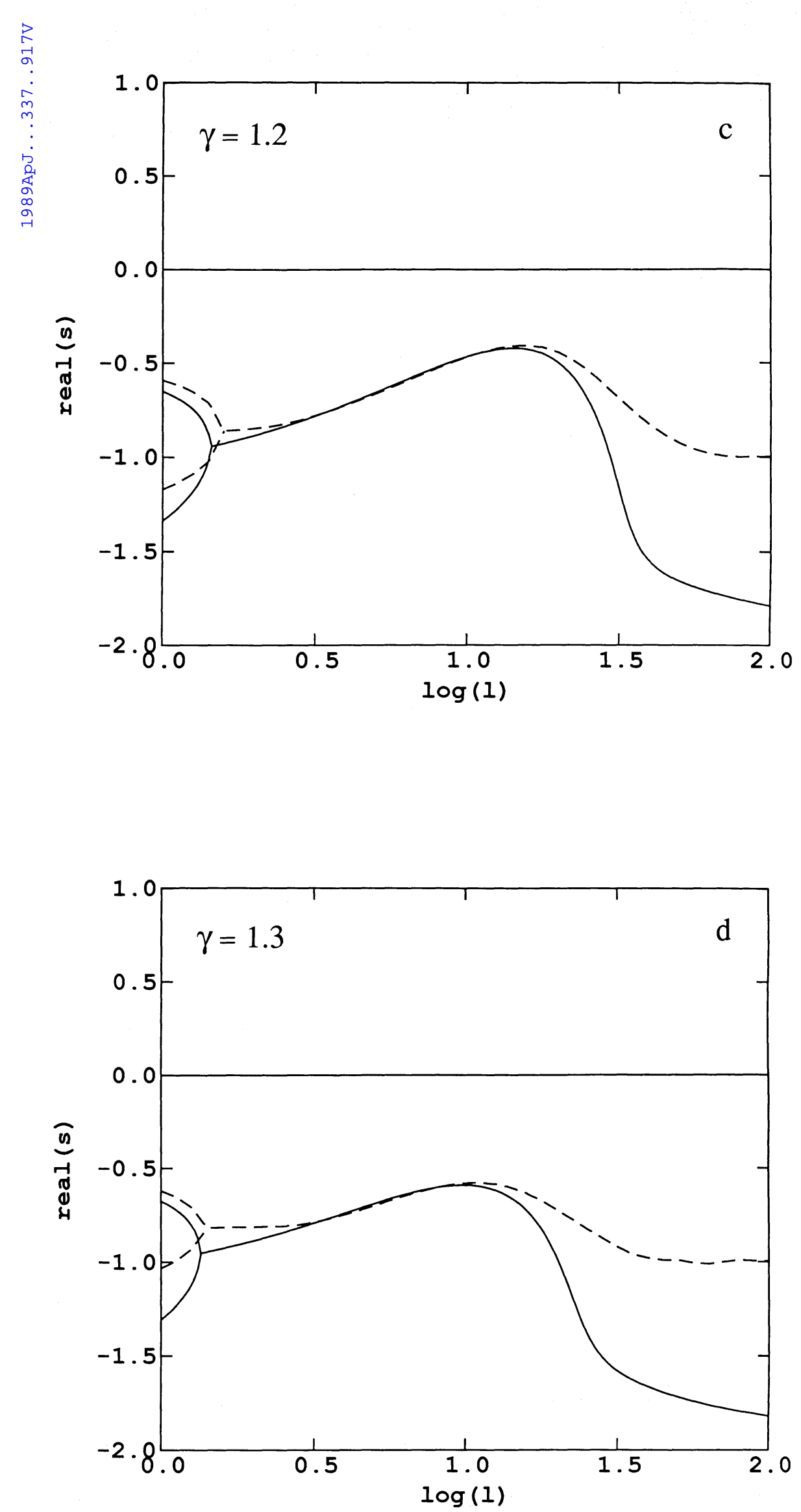

FIG. 2-Continued 
underestimated by considering only the value of the pressure in the absolute center of the blast.

We can repeat this procedure for the wind-driven shock where $M=0.6$, and $\beta \approx \frac{7}{9}$. Exact values of $\beta$ for varying values of $\gamma$ are contained in Table 1. Repeating our calculations in this case we find marginal stability for $\gamma=1.0995$. For $\gamma=1.05$ we find instability if $15.8<l<93.3$. Numerical work (Ryu and Vishniac 1988) suggests marginal stability when $\gamma \approx 1.1$ and instability for $15<l<100$ when $\gamma=1.05$. Figure 2 shows the comparison between our estimates of $\Re s$ for $\gamma=1.05,1.1$, and 1.3 , and the calculations of the exact values. In this case the only substantial deviation is for very high frequency perturbations. In general the agreement is better in this case than in the blast wave case. The reason is that the entropy of the postshock gas is a more slowly varying function of the masss shell in the wind-driven case. Therefore we expect our exact results to be better approximated by an isothermal treatment.

We are now in a position to evaluate the stability of other self-similar shocks with confidence. In particular we can use our results to estimate the stability of isothermal shocks (which is what they were intended for). We consider the ideal isothermal shock with spherical symmetry expanding into a uniform medium and driven by residual gas pressure at the core. In this case, $M=\frac{2}{7}$, and $\beta=\frac{1}{6}$. We find marginal stability for $\mathscr{M}=3.27$ (or a density contrast of $\sim 10$ ). For $\mathscr{M}=5$ we have instability for $7.77<l<115$. We note that this instability will not appear in computer simulations unless numerical effects are mild enough to allow density contrasts greater than 10 . We show the predicted values of $\Re s$ for $\mathscr{M}=5$ in Figure 3.

The related case of an isothermal shock driven by a wind has $M=0.6$, and $\beta=\frac{7}{9}$. We find marginal stability for $\mathscr{M}=4.95$.
TABLE 1

Parameter $\beta$ For the Adiabatic WIND-DRIVEN SHOCK

\begin{tabular}{cc}
\hline \multicolumn{1}{c}{$\gamma$} & $\beta$ \\
\hline $1.05 \ldots \ldots \ldots \ldots \ldots \ldots$ & 0.7579 \\
$1.1 \ldots \ldots \ldots \ldots \ldots \ldots \ldots$ & 0.7391 \\
$1.2 \ldots \ldots \ldots \ldots \ldots \ldots \cdots$ & 0.7050 \\
$1.3 \ldots \ldots \ldots \ldots \ldots \ldots$ & 0.6751 \\
\hline
\end{tabular}

For $\mathscr{M}=7$ we have instability for $16.1<l<111$. We show the predicted values of $\mathfrak{R}$ for $\mathscr{M}=7$ in Figure 4 .

\section{SUMMARY AND CONCLUSIONS}

We have derived a general treatment for linear perturbations of shock waves. Our motivation is quite simple. We wish to demonstrate that the gross dynamics of shock waves can be understood in simple terms without reference to the details of their postshock structure. This is an idea that has been previously explored for the long-wavelength perturbations of shocks (V83) and the evolution of one-dimensional shocks (Ostriker and McKee 1988). Here we have extended it to the full range of linear perturbations of shocks. Our approximate treatment requires knowledge of just a few global parameters of the shock wave, i.e., the shock speed, the mass surface density, the external density, the interior pressure, and some appropriately weighted sound speed in the dense postshock gas. The dynamical equations are (15a), (15b), and (17). In the limit of strong instability, when the secular evolution of the shock may be neglected, we find the dispersion relation given

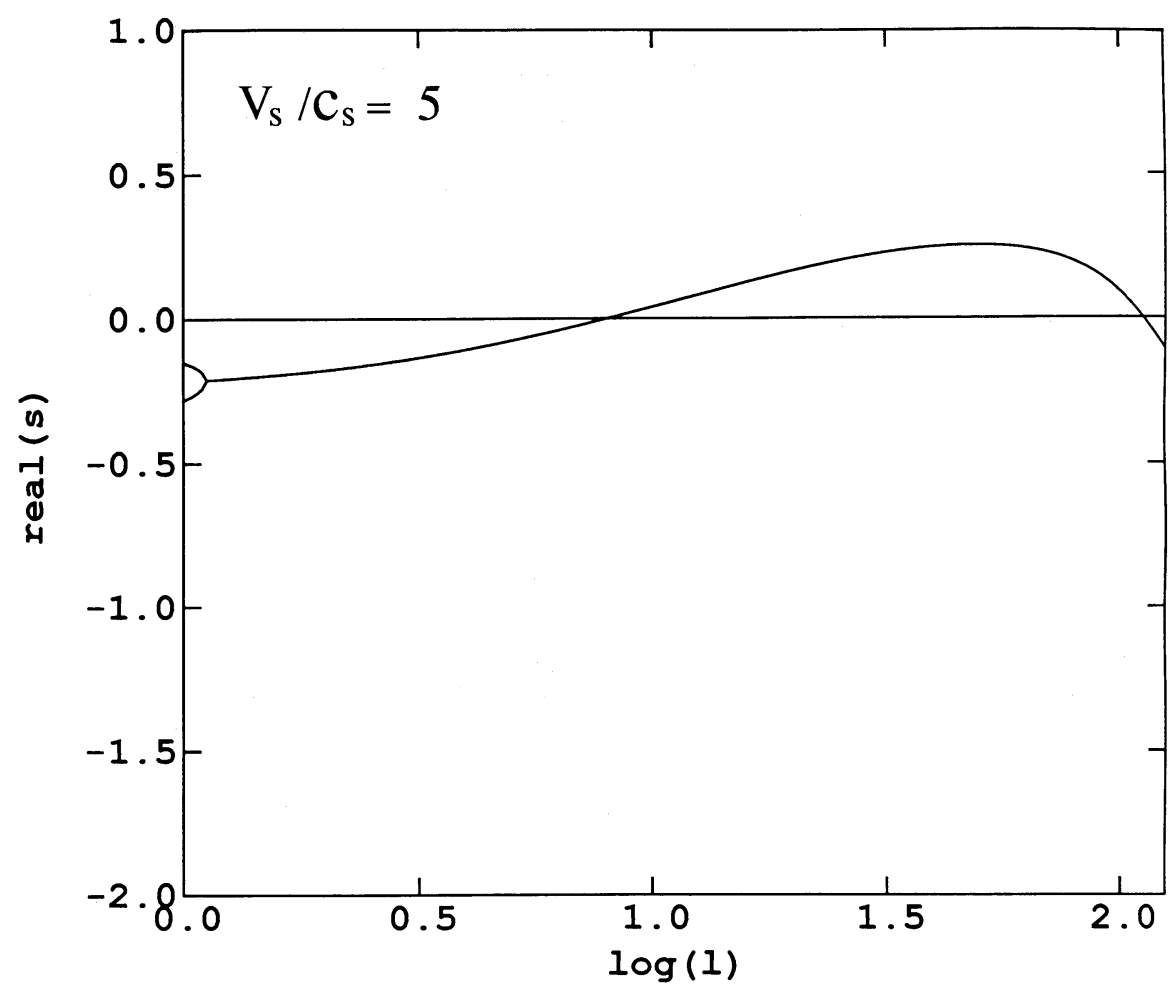

FIG. 3.-A plot of the dimensionless growth rate, real(s), as a function of spherical eigennumber for perturbations of an isothermal blast wave with $\mathscr{M}=5$. The curve plotted is the prediction of the approximate treatment given in the text. We expect it to be accurate to order $\mathscr{M}^{-2}$. 


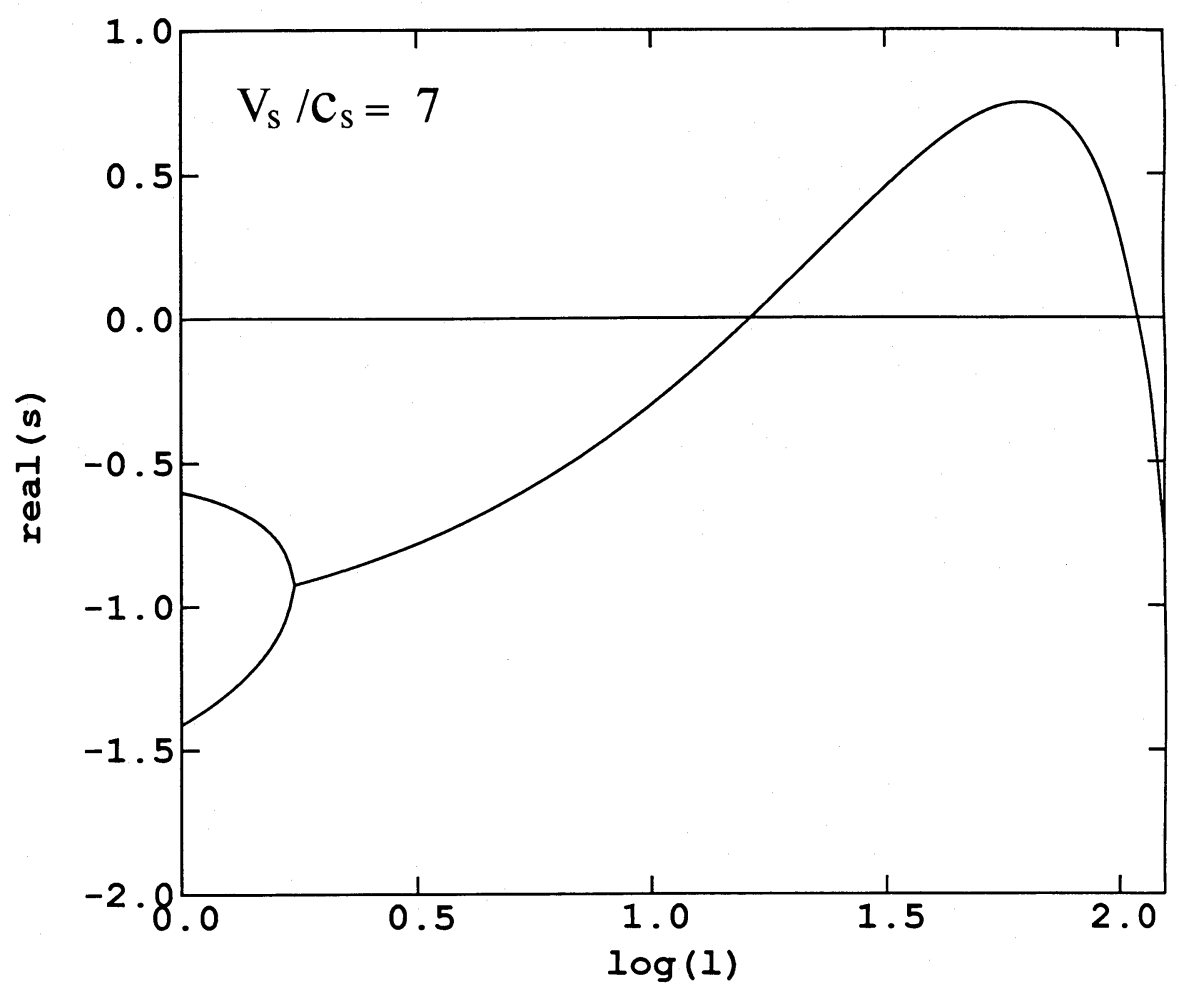

Fig. 4.-A plot of the dimensionless growth rate, real(s), as a function of spherical eigennumber for perturbations of an isothermal wind driven shock with $\mathscr{M}=7$. The curve plotted is the prediction of the approximate treatment given in the text. We expect it to be accurate to order $\mathscr{M}^{-2}$.

in equation (13). This point of view does have its limits. For example, we cannot comment, using this formalism, on the existence of thermal instabilities (for a discussion see Chevalier and Imamura 1982 and references therein). It is not difficult to understand why the overstable modes are relatively insensitive to the details of the postshock structure. These modes are fundamental modes in the sense of having no radial (i.e., perpendicular to the shock front) nodes. In general, modes with sufficiently long wavelengths in some direction will only be sensitive to the structure of the system averaged over the direction of maximum wavelength.

We note in passing that this approach to the dynamics of an isothermal shock can be adapted to examine the gravitational instabilities of such shocks. However, we expect that the results will be of physical interest only when the dynamical instability described here is ineffective, i.e., when $\beta=0$, or $\dot{V}_{s}=0$, or when the value of $\mathscr{M}$ is relatively small. Otherwise the details of the gravitational modes will be of purely academic interest. The real physical problem will be one of the aggregation of separate fragments of the shock wave.

We have tested our approach through comparisons with previous numerical work. Although these tests are limited to comparisons with spherically symmetric, adiabatic cases, the quality of agreement with our approximate results gives us reason to believe that our formulae are generally accurate and can be used to evaluate the stability of any realistic shock whose evolution is given from a one dimensional numerical model. We have applied this work to the case of the perfectly isothermal blast wave and conclude that it will be unstable if the density contrast in the shell exceeds $\sim 10$. The related case of an isothermal shock driven by a wind will be unstable if the density contrast exceeds $\sim 25$.

This work has been supported in part by NSF grant AST8451736 .

\section{REFERENCES}

Bernstein, I. B., and Book, D. L. 1987, Ap. J., 225, 633.

Bertschinger, E. 1986, Ap. J., 304, 154.

Chevalier, R. A., and Imamura, J. 1982, Ap. J., 261, 543.

Erpenbeck, J. J. 1962, Phys. Fluids, 5, 1181 .
Ostriker, J. P. and McKee, C. 1988, Rev. Mod. Phys., 60, 1. Ryu, D., and Vishniac, E. T. 1987, Ap. J., 313,841. Vishn. 1988, Ap. J., 331, 350.

Vishniac, E. T. 1983, Ap. J., 274, 152 (V83).

EThAN T. VishniAC and DongSU RyU: Department of Astronomy, University of Texas, Austin, TX 78712 